البيئة القديمة لتكوين كراكو والجزء العلوي من تكوين رطاوي (الكريتاسي الاسفل) من وسط العرلق

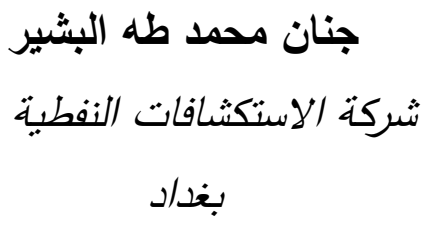

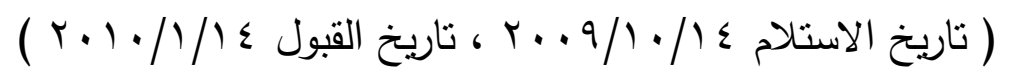

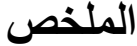

تمت دراسة حشود أجناس الاوستراكودا من تكويني كراكو ورطاوي (الكريتاسي الاسفل)، من آبار

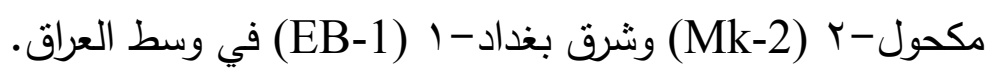

حددت البيئة القديمة لنكويني كراكو ورطاوي استتادا الى مصنفات اجناس الاوستراكودا المترافقة (الاجناس) في الابار مكحول-r وشرق بغداد-1 وضمن نماذج اللباب الصخري فقط، فظهر بان تكوين كراكو يمثل بيئة بحرية ضحلة ضمن منطقة (sublittoral)، ذات ملوحة بحرية اعتبادية، ومياه دافئة، اما نكوين رطاوي فظهر بانـه ترسب في بيئة بحرية ضحلة قد تصبح (inner platform) كما توجد دلائل تكوين البيئات السامة ذات المحتوى الاوكسجيني القليل.

\title{
Paleoecology of Garagu Formation and the Upper Part of Ratawi Formation (Lower Cretaceous) of Central Iraq
}

Sanad A. Al-Khashab

Department of Geology

College of Science

Mosul University
Salih K. Khalaf

Department of Geology

College of Science

Mosul University
Jinan M. Al-Basher

Oil Exploration Company

Baghdad

\section{ABSTRACT}

Ostracode assemblages from Garagu and Ratawi Formations (Lower Cretaceous) from Makhul-2 (Mk-2) and East Baghdad-1 (EB-1) boreholes were studied in details, leading to the conclusion that Garagu Formation deposited under shallow marine sublittoral environment with normal salinity and warm water, while Ratawi Formation deposited in shallow marine environment with anoxic environments. 


\section{المقدمة}

أخذت نماذج الدراسة الحالية من الابار مكحول-r (Mk-2) وشرق بغداد-1 (EB-1) في وسط

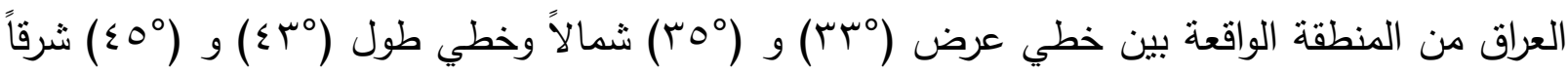
( شكل ( )، والموضح تفاصيلها في ( الثكل r ) و (الثكل r)، مثلت هذه النماذج تكويني راكو ورطاوي.

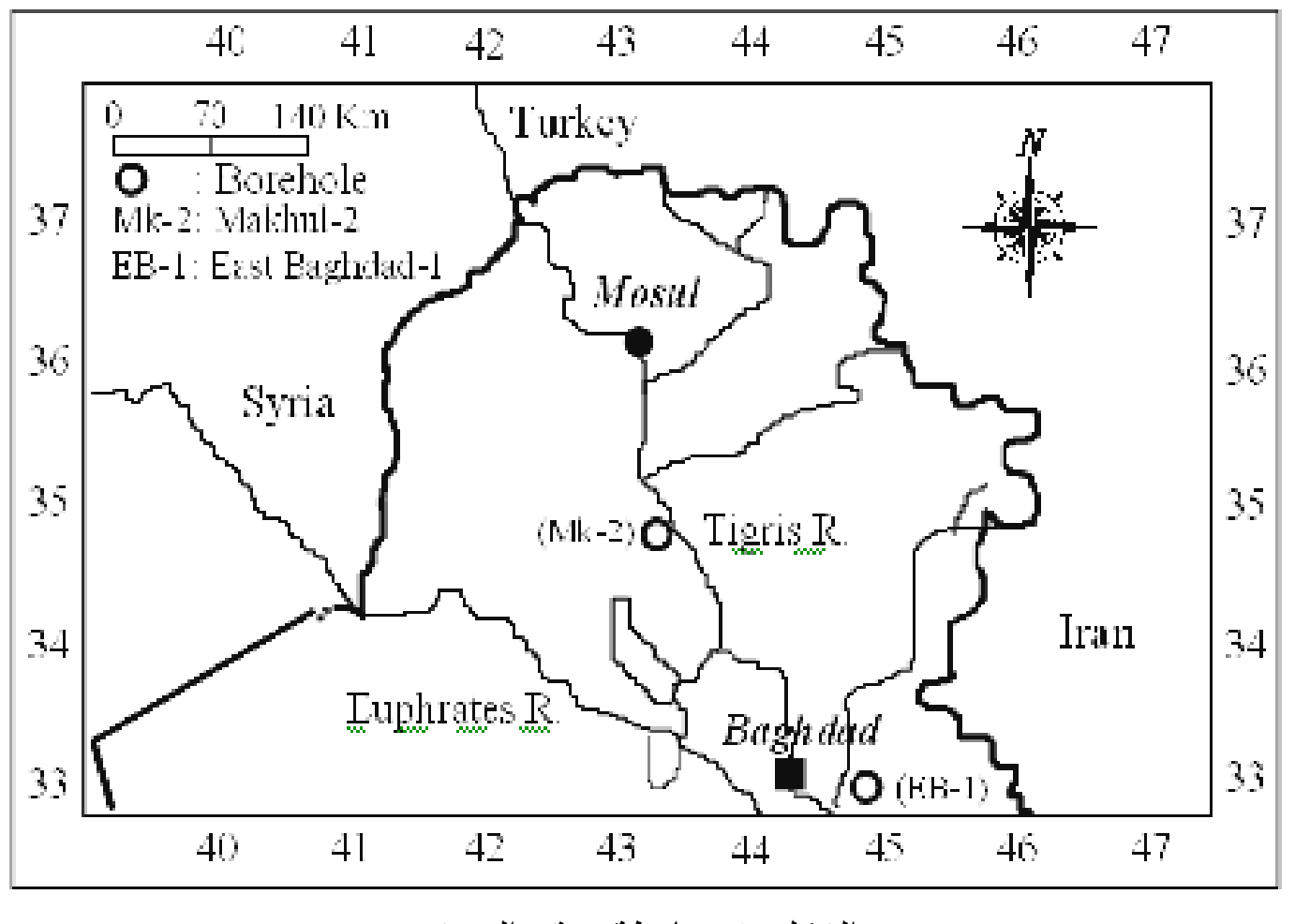

الثكل ا : خارطة موقع البحث

ذكر (Chatton and Hart, 1960) بان تكوين كراكو يتكون بشكل رئيسي من الحجر الجيري

الفتاتي العضوي وحجر الرمل والسجيل والكالكرينايت، وانه يمثل سحنة وسطية لتكويني يمامة وزبير • بينما اوضح التقرير النهائي لبئر سامراء-1 (Johnson, 1961) الى ان تكوين كراكو كان ترسب في مياه ضحلة وانه يتكون من حجر جيري ذا سرئيات كاذبة ودمالق ويحوي جزئياً الفتاتيات واحياناً متدلمتة فضلاً عن السجيل الرملي. واشار (Ditmar et al., 1971) إلى أن البيئة الترسيبية لتكوين كراكو تتمنل بالبيئة البحرية الثاطئية مع مزيج من المواد الفتانية والحجر الجيري السرئي بصورة رئيسية. وكان Bartenstein) Jassim أثارا إلى أن تكوين كراكو ترسب في بيئات البحيرات الشاطئية، ايضا and Abawi, 1984) (and Karim, 1984) ان التكوين ترسب في مياه ضحلة مدارية الى شبه مدارية ذات ملوحة اعتيادية الى عالية، ومنمثلة ببيئة المنصة ومنطقة المنصة المعزولة. واشارت الدراسات المقدمة من قبل شركتي British) Petroleum and Idemitsu, 1990) 
مفتوحة ذات انغلاق جزئي ومياه ذات طاقة عالية. وبين (Al-Eisa, 1997) بان التكوين يمنل سحنات

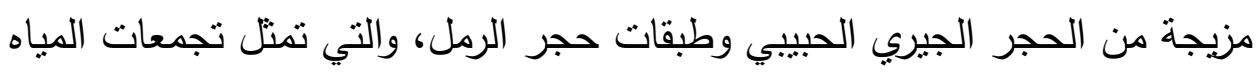
الضحلة.أثنارت دراسات (Bellen et al., 1959) الى ان الجزء العلوي من تكوين رطاوي يتكون من حجر التهري السجيل الاسود الدخضر والحاوي على البايرايت، وانشاروا الى ان الجزء العلوي من التكوين مكافئ طباقيا للجزء السفلي من تكوين زبير في ابار وسط العراق، ولم يشر الباحثون الى بيئة الترسيب لتكوين رطاوي

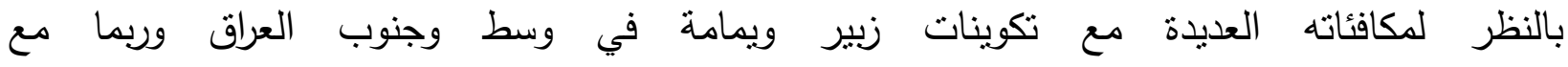
كراكو في شمال العراق، بينما اثشار (Douban and Medhadi, 1999) إلى أن الجزء العلوي من تكوين رطاوي والمتكون من حجر السجيل shale ترسب في بيئة ذات اعماق تصل بين inner shelf الى فيل .middle shelf

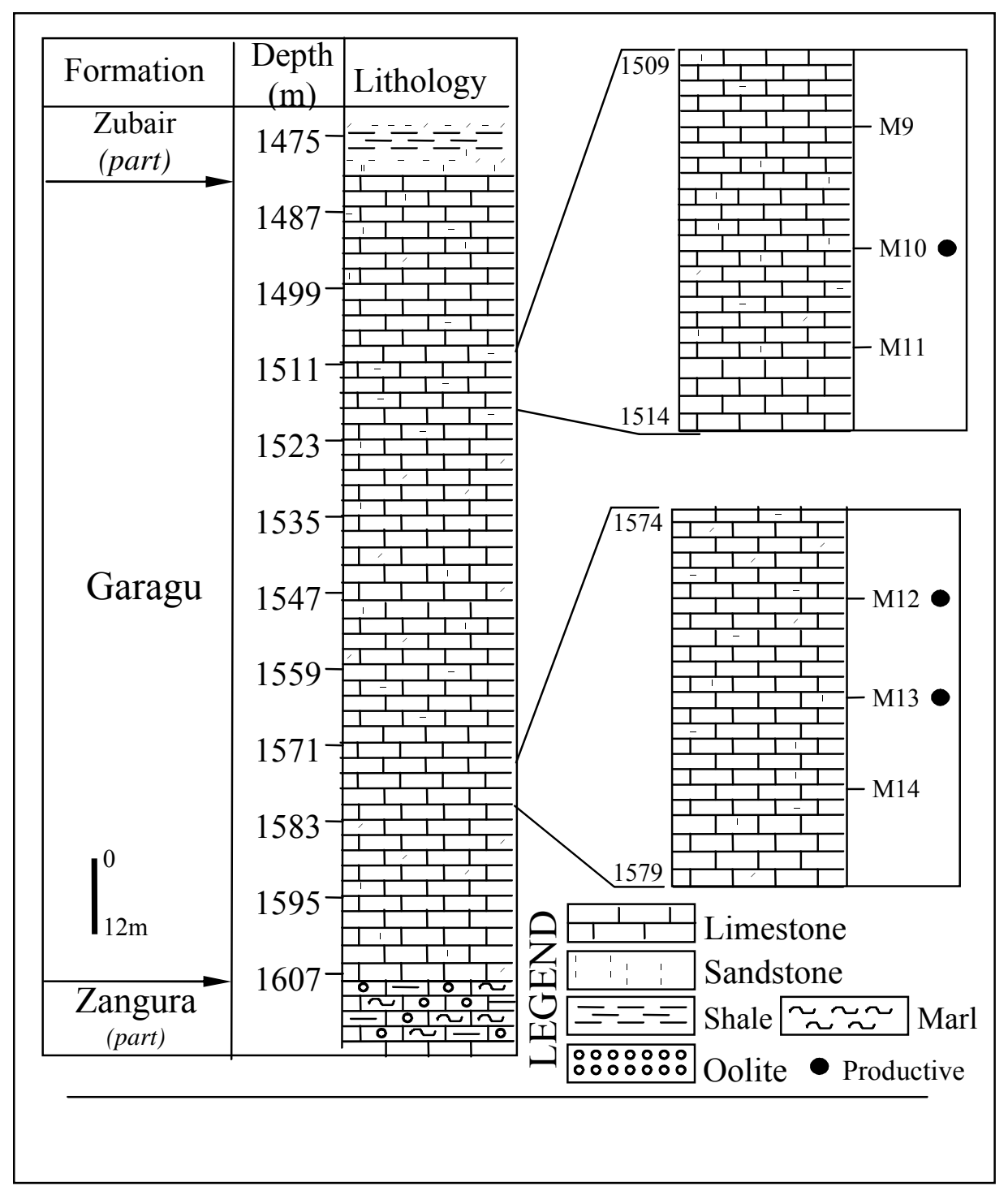

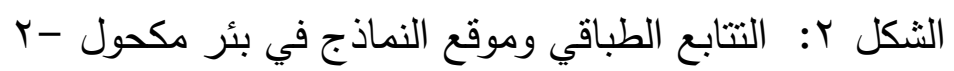




\section{النمذجة وموقع الدراسة}

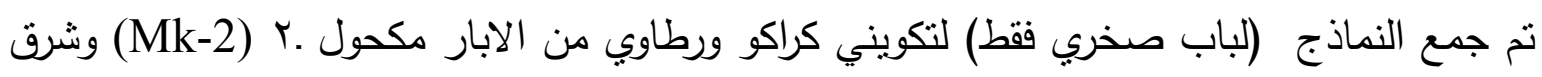

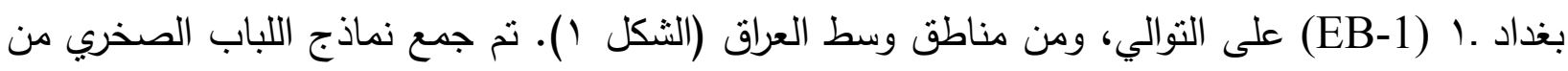

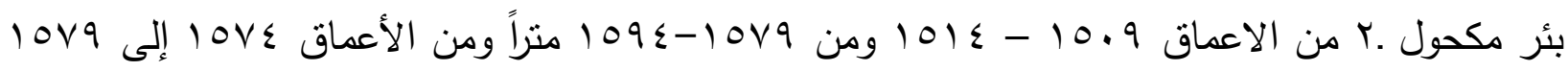

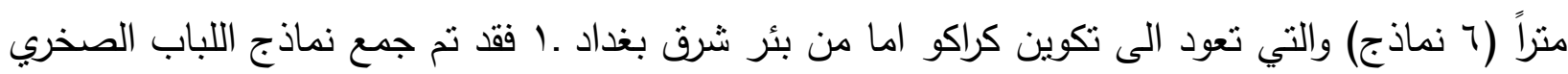

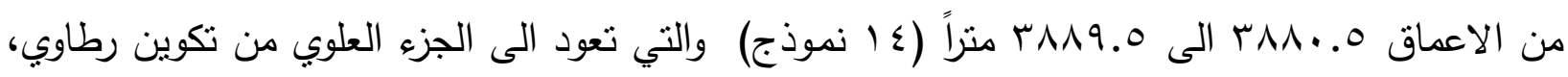
و (الجدول () يوضح آبار الدراسة وسمك كلا التكوينين حسب النقارير النهائية لثركة الاستكثافات النفطية، وعدد نماذج اللباب الصخري المأخوذة منها.

الجدول ا: سمك التكوينات وعدد النماذج في الآبار قيد الدراسة.

\begin{tabular}{|c|c|c|c|c|}
\hline \multicolumn{2}{|c|}{ شرق بغداد 1} & \multicolumn{2}{|c|}{ مكحول r } & \\
\hline عدد النماذج & السمك (متر) & عدد النماذج & السمك (متر) & \\
\hline & & 7 & $1 K 9$ & كراكو \\
\hline $1 \varepsilon$ & 090 & & & رطاوي \\
\hline
\end{tabular}

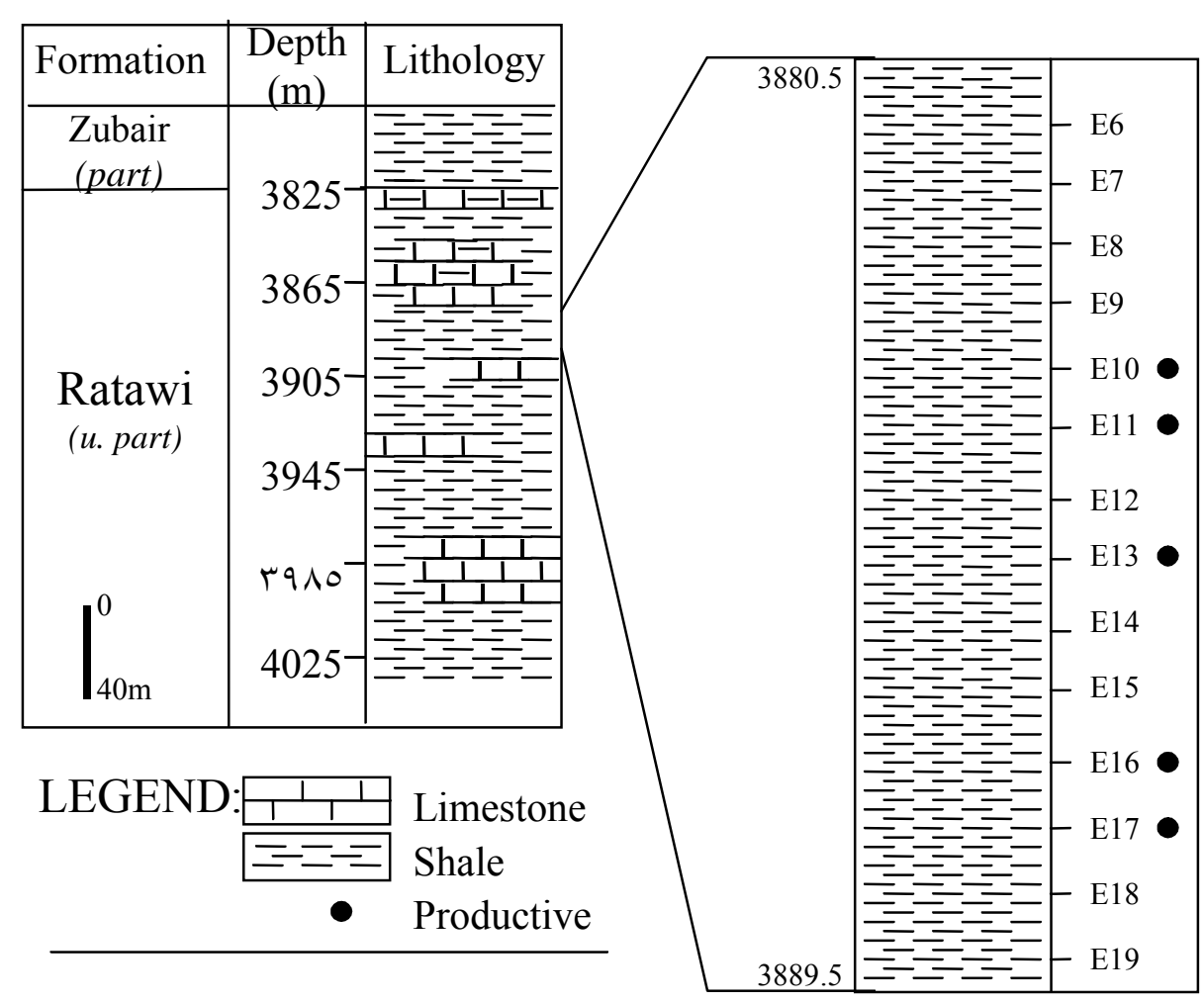

الثكل rا: التتابع الطباقي وموقع النماذج في بئر شرق بغداد - 
البيئة القديمة لنكوين كراكو والجزء العلوي من تكوين رطاوي (الكريناسي الاسفل)...

الدراسة التصنيفية

بعد معالجة النماذج مختبريا تم التقاط عينات الاوستراكودا ومن ثم تصنيفها بالاعتماد على التصانيف

العالمية والدوريات الحديثة. حيث اعتمد تصنيف (Morkhoven, 1962, 1963) وكذلك نم استخدام

تصنيف (Hartmann and Puri, 1974)، أما المظاهر الخاصة بالزخرفة فقد اعتمد في وصفها على (V)

(Sylvester-Bradley and Benson, 1971)

الدوريات والنشرات الحديثة التي تم من خلالها التعرف على الاجناس والانواع الجديدة والموصوفة ما بعد عام ع ا9 ا ـ وفيما يلي قائمة بالأنواع والنماذج التي ظهرت فيها والتكوين الذي وجدت فيه:

1- Cytherella ovata (Römer) Jones 1849, P1.1 , Fig.1, samples E11, E17, Ratawi Fm.

2- Cytherella posterosulcata Al-Abdul-Razzaq 1981, Pl.1, Figs.2-3, sample E11, Ratawi Fm.

3- Cytherella cf. sp. OUM1110 Grekoff 1969, Pl.1 , Fig.4, sample E10, Ratawi Fm.

4- Cytherella sp. Damotte, Zghal and Bismuth 1987, Pl.1, Fig.5, sample M10, Garagu Fm.

5- Cytherella sp. U10 Glintz and Magne 1959, Pl.1, Fig.6, sample E11, atawi Fm.

6- Paracypris? sp., Majoran 1989, P1.1 , Fig.7, sample M10, Garagu Fm.

7- Macrocypris aff. siliqua (Jones 1849), P1.1, Fig.8, sample E13, Ratawi Fm.

8- Asciocythere cf. circumdata (Donze) Andreu et al., 1996, Pl.1, Figs.9-10, sample E16, Ratawi Fm.

9- Asciocythere sp.1 Babinot et al. 1985, P1.), Fig.11, samples E11, E13, Ratawi Fm.

10- Schuleridea cf. altera Kuznetsova 1961, Pl.1, Figs.12-13, samples E11, 17, Ratawi Fm.

11- Schuleridea cf. obtabilis Kuznetsova 1961, Pl.1, Figs.14-15, sample M10, Garagu Fm.

12- Metacytheropteron? grosdidieri Damotte, Zghal and Bismuth, 1987, Pl.1, Fig.16, samples M12, Garagu Fm.

13- Cythereis btaterensis btaterensis Bischoff 1963, P1.1, Fig.17, sample M12, Garagu Fm.

14- Cythereis laqueformis Bischoff 1963, P1.1, Figs.18-20, samples M12, M13, M16, Garagu Fm.

15- Rehacythereis bernardi Grosdidier 1964, P1.1, Fig.21, sample M12, Garagu Fm.

16- Rehacythereis cf. libanensis (Bischoff 1963), P1.1, Fig.22, sample M12, Garagu Fm.

17- Spinoleberis yotvataensis Rosenfeld in Rosenfeld and Raab 1974, Pl.1, Fig. 23, sample M12, Garagu Fm. 


\section{البيئة القديمة}

تستخدم الاوستراكودا في التفسيرات البيئية القديمة، حيث انشار (Mazzini et al,. 1999) و (Elewa, 2004) الى أن الاوستراكودا تعطي دلائل ممتازة في التفسيرات البيئية القديمة والحديثة، وتتير بعض اجناس الاوستراكودا الى بيئة معينة بينما البعض الاخر يدل على مدى واسع من البيئات وبعضها يفسر عن طريق وجوده مع اجناس اخرى مرافقة، وادناه استعراض للاجناس ذات البيئة المعروفة والتي

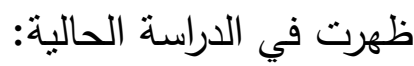
Paracypris جيد من الاوكسجين مع محتوى عالٍ من CaCo3. (Van Morkhoven, 1963) يوضح أن أن bathyal infra neritic ذو بيئة بحرية وبصورة رئيسية تكون عميقة من Paracypris (الثكل \&)، كما انشار (Dall'antonia et al., 2003) إلى أن تجمعات اوستراكودا المياه العميقة ومن ضمنها Paracypris تتميز بكونها نحيفة وملساء. (Zarraga and Lazaro, 1990) :Schuleridea .inner-platform Cythereis .(Bassoullet and Damotte, 1969) Rehacythereis .(Lazaro, 1988) كما فسر العديد من الباحثين بيئة الترسيب القديمة استتادا الى تواجد الاجناس على اساس ترافقها مع

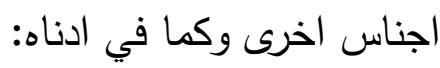

التفسيرات البيئية باستخدام تجمعات الاوستراكودا

الباحثن في مجال البيئة القديمة لا يفسرون بيئة الترسيب لتكوين معين بمعرفة بيئة جنس معين فقط بل ايضا يعتمدون على اساس ترافق مجموعة اجناس (اثنان فأكثر)، حيث ان الاوستراكودا نتواجد في مدى

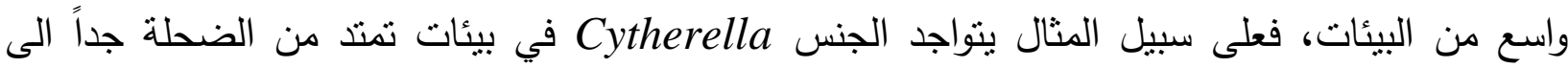
العميقة جداً ويمكن ان يتواجد ايضاً في البيئات السامة (ذات المحتوى الاوكسيجيني الردىء)، وعليه فان استخدامه في التفسيرات البيئية لوحده يعطي نتائج خاطئة، لذلك تتم التفسيرات البيئية على اساس الترافق ل لهجاميع الاوستراكودا حيث يعطي ذلك نتائج جيدة. إن العثور على الجن Cytherella بوفرة مرافقاً للجنس Cythereis يشير الى ظروف مياه ضحلة جداً (Rosenfeld and Raab, 1974). (Breman, 1976) أثنار بان بورة التجمع Rardoppilata و و Rachacythereis و Cytherella 


\section{البيئة القديمة لتكوين كراكو والجزء العلوي من تكوين رطاوي (الكريتاسي الاسفل).....................}

Cytherelloidea, Cytherella, إلاغلب مياه عميقة. بينما اثار (Neale, 1977) إلى إن ترافق (Zarraga and Lazaro, 1990) يدل على تجمع مياه دافئة. Paracypris, Bairdia, Schuleridea بينّا بان وجود Cytherella مع Outocyprella يشير الى ظروف Outer-platform (الثكل \&). Cytherella, Paracypris, وجدوا تجمع من الاوستراكودا يضم لونم Asciocythere, Dolocytheridea

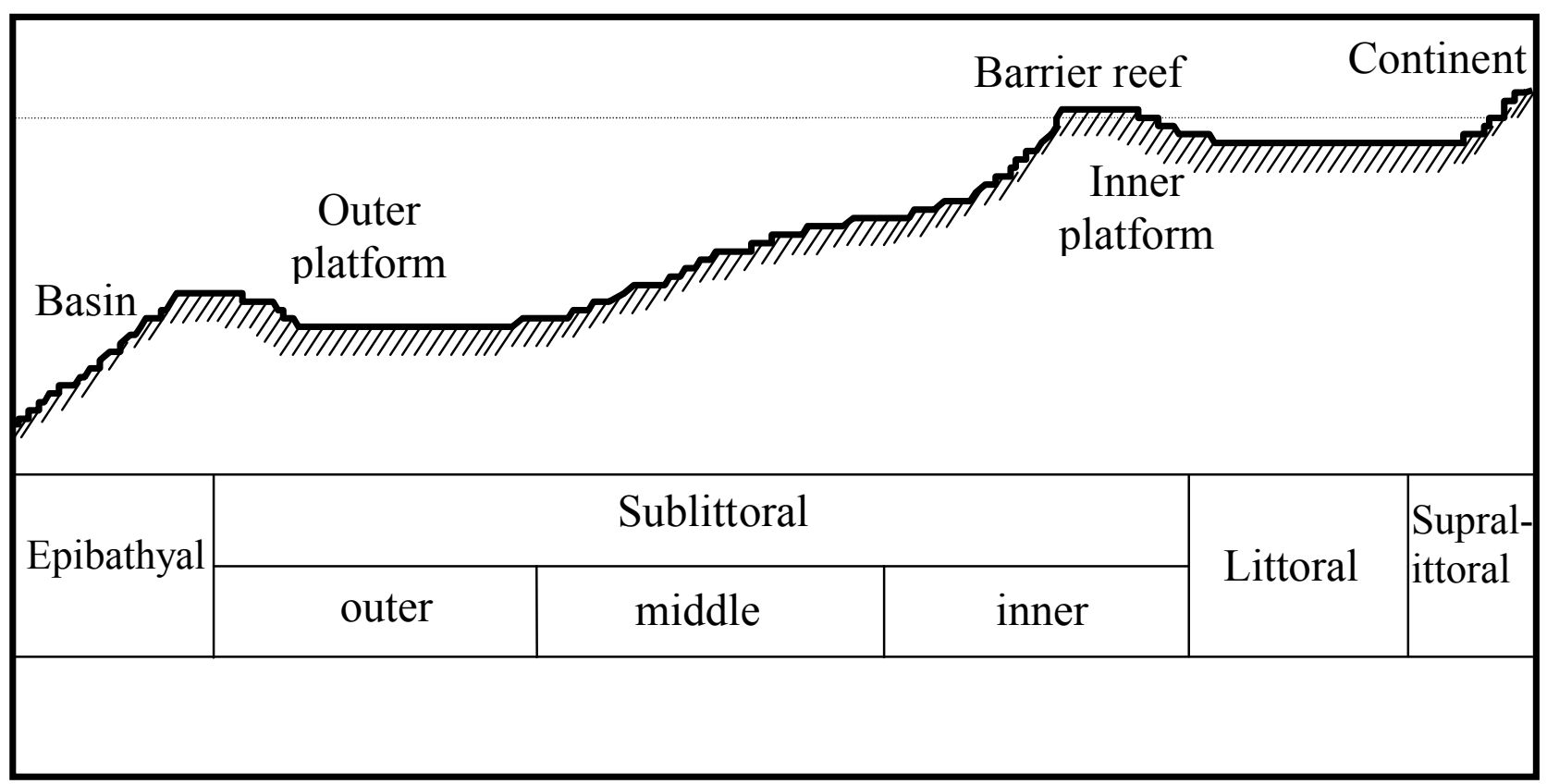

الثكل ؛؛: تقسيمات الحوض الترسيبي (Kuznetsova and Dobrova, 1997)

Cytherella, Metacytheropteron, Veeniacythereis, Dolocytheridea, ترافق الاجناس Eocytheropteron, Bairdia Cytherella, Bairdia, Eocytheropteron, Dolocytheridea, g Cythereis g Glenocythere Paracypris (Warne, 1993) بان وجود Cytherelloidea , Cytherella مع اجناس اخرى يشير الى بيئات .mid-shelf

بينما المجموعة التي تضم Cytherella مع Bairdia واجناس اخرى نتير الى مياه عميقة. وجدوا اجناساً منها Schuleridea, Cytherelloidea, Cytherella, واشناروا (Malz et al., 1985)

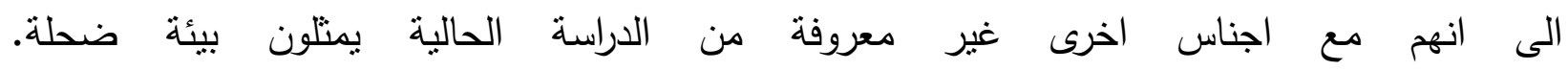
(Ishizaki and Kaiho 199•) 
Bairdia

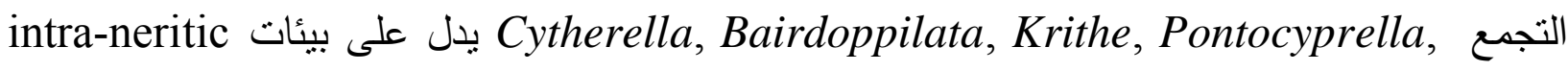
الى bathyal

كما أثنار (Warne, 1993) إلى أن المجموعة التي تضم Cytherella مع Bairdia واجناس

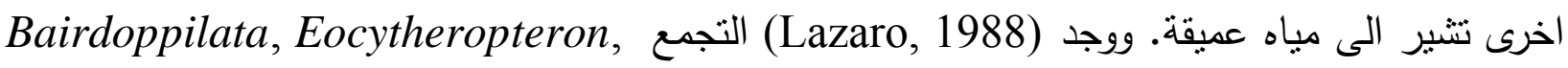
Schuleridea, Rehacythereis (Serissocytheridea ذكروا بان المجموعة التي تتميز بوجود (Szczechura et al., 1991) Candoniella خلال الترسيب كان هناك دخول للمياه العذبة وهذا قد ينتج بسبب الفعاليات التكتونية والتي شخصت خلاهل الكريتاسي الاوسط في جنوب التيش من قبل (Abdul-Razzaq and Grosdidier, 1981) وقبلت من

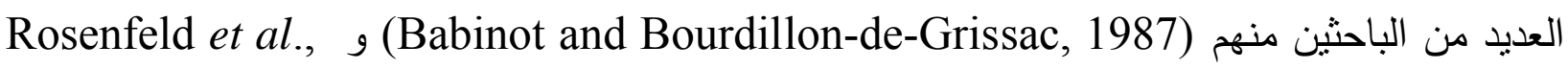
Rehacythereis, Pontocyprella, بين بان التجمع (Zarraga and Lazaro, 1990) (1988) .open-platform يشير الى ظروف Neocythere Cytherella, Bairdoppilata, Krithe, Pontocyprella, التجمع (Dingle, 1981) وجنع

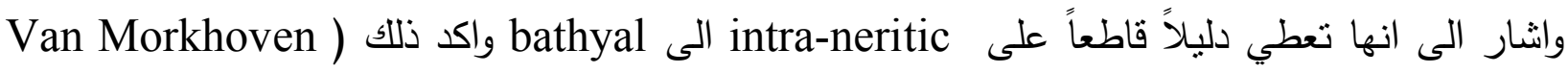

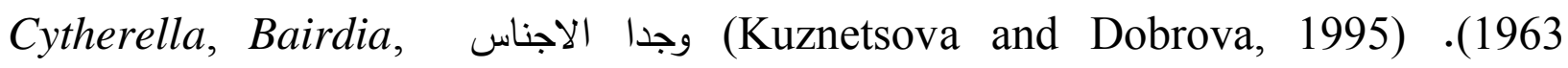
Macrocypris يضم Dolocytheridea و Rehacythereis و Asciocythere يمثل بيئات بحرية ضحلة او مختلطة. بينما وجد (Andreu et al., 1996) تجمعاً يحوي Schuleridea و و و Schuleridea محصورة، كما بينوا بان التجمع Dolocytheridea

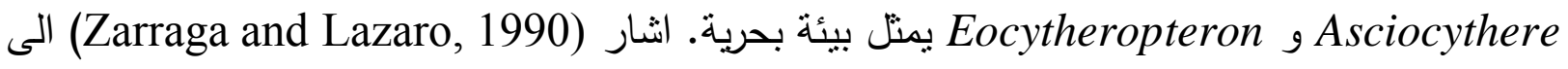

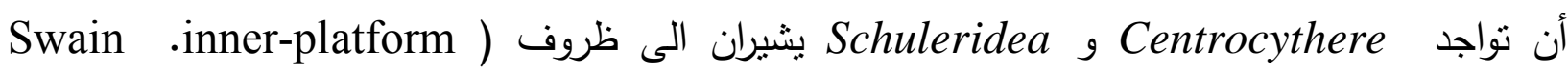
1985) وجد اجناس مثل Rehacythereis و واجد و Echuleridea وانشار الى انهم

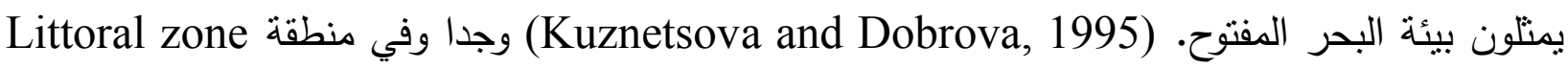
(الثكل §) التجمع الذي يضم اجناس مثل Schuleridea, Cytherelloidea. مما تقام من استعراض لبيئة الاجناس التي ظهرت في الدراسة الحالية، ومن خلال محتوى كل تكوين

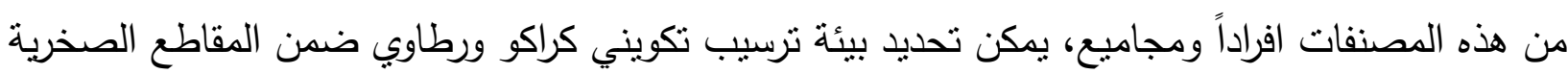
(اللباب الصخري) الخاصة بها. (الجدول r) يوضح مصنفات الاوستراكودا (على مستوى الجنس) التي وجدت في التكوينين قيد الدراسة وضمن النماذج (M10, M12, M13) في بئر مكحول-r لتكوين كراكو وضمن النماذج 
(E10, E11, E13, E16, E17) التعرف على الييئة القديمة من خلال دراسة ترافق مجاميع الاوستراكودا ضمن النماذج قيد الدراسة. يحتوي النموذج (M13) وهو الاعمق على الجن Cythereis متمثلا بالنوع Cythereis فقط وهو يشير لوحده طبقاً الى (Swain, 1982) الى ظروف مياه ضحلة،

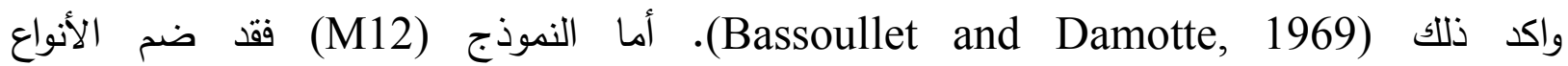
Metacytheropteron? ,Cythereis laqueformis , Cythereis btaterensis btaterensis Spinoleberis yotvataensis و Rehacythereis bernardi grosdidieri الباحثين الى هكذا ترافق ولكن ظهور الجن Cythereis في هذا النموذج يشير الى ظروف مياه ضحلة ( Swain, 1982; Bassoullet and Damotte, 1969)

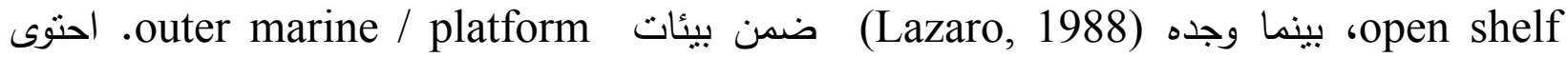
Schuleridea cf. و Paracypris? sp و Cytherella sp على الانموذج (M10) عنى obtabilis وهذا التجمع يختلف كليا عن النموذجين السابقين حيث اثار (Neale, 1977) الى ان هذا التجمع اضافة الى Bairdia يثير الى الترسيب في المياه الدافئة. كما وجد (Malz et al., 1985) نفس

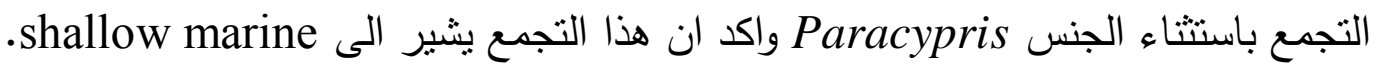

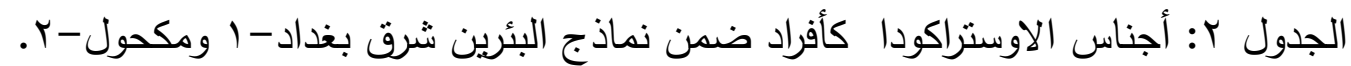

\begin{tabular}{|c|c|c|c|c|c|c|c|c|c|}
\hline \multirow{3}{*}{ Genera } & Sample No. & E10 & E11 & E13 & E16 & E17 & M10 & M12 & M13 \\
\hline & Depth (m) & $\begin{array}{l}3883.5 \\
3887.1 \\
\end{array}$ & \begin{tabular}{|l}
3883.5 \\
3887.1 \\
\end{tabular} & \begin{tabular}{|l|l|}
3883.5 \\
3887.1
\end{tabular} & $\begin{array}{l}3887.1- \\
3889.5\end{array}$ & \begin{tabular}{|l}
3887.1 \\
3889.5 \\
\end{tabular} & $\begin{array}{l}1509.7- \\
1510.3\end{array}$ & \begin{tabular}{|l|}
$1511.5-$ \\
1512.1
\end{tabular} & \begin{tabular}{|l}
$1512.4-$ \\
1513
\end{tabular} \\
\hline & Formation & \multicolumn{5}{|c|}{ Ratawi } & \multicolumn{3}{|c|}{ Garagu } \\
\hline \multicolumn{2}{|c|}{ Asciocythere } & & 3 & 2 & 4 & & & & \\
\hline \multicolumn{2}{|c|}{ Cytherella } & 4 & 17 & & & 4 & 5 & & \\
\hline \multicolumn{2}{|c|}{ Cythereis } & & & & & & & 7 & 2 \\
\hline \multicolumn{2}{|c|}{ Macrocypris } & & & 3 & & & & & \\
\hline \multicolumn{2}{|c|}{ Metacytheropteron } & & & & & & & 4 & \\
\hline \multicolumn{2}{|c|}{ Paracypris } & & & & & & 5 & & \\
\hline \multicolumn{2}{|c|}{ Rehacythereis } & & & & & & & 4 & \\
\hline \multicolumn{2}{|c|}{ Schuleridea } & & 2 & & & 2 & 3 & & \\
\hline \multicolumn{2}{|c|}{ Spinoleberis } & & & & & & & 3 & \\
\hline
\end{tabular}


ضم النموذج (E17) الانواع Schuleridea cf. altera , Cytherella ovata اللذان يشيران الى البيئات البحرية الضحلة وربما الدافئة ان وجد الجنس Cytherelloidea مرافقاً لهم Asciocythere cf. والنموذج (Malz et al., 1985; Neale, 1977) circumdata

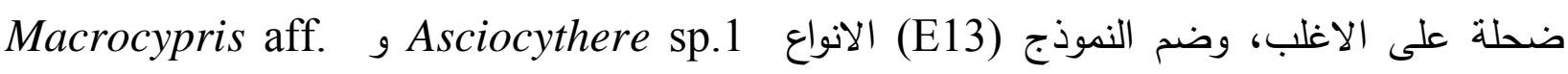

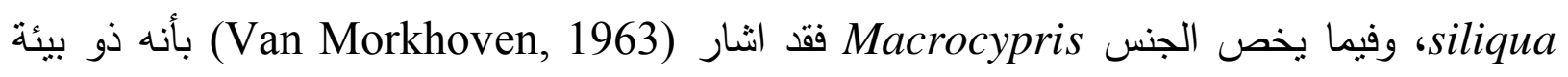

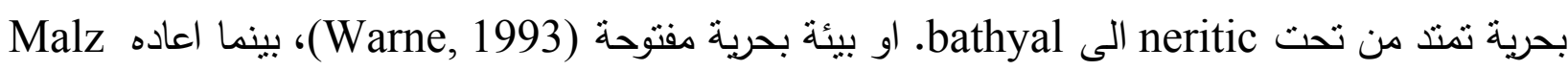
(et al., 1985)

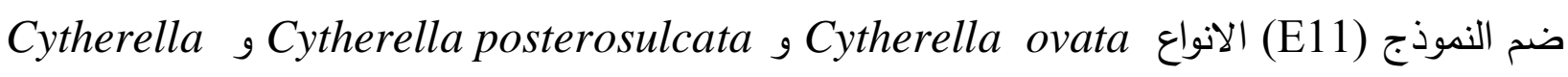
inner- ويثير نواجد الجنس Schuleridea cf. altera و و والى ظروف Sch. U10

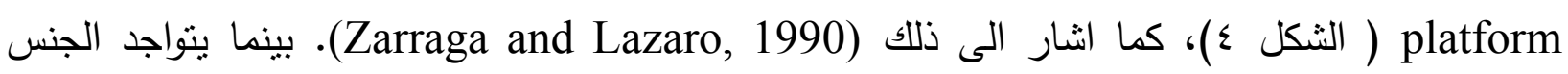
Cytherella inner-platform يمكن من خلاله وحده الاستدلال على البيئة الترسيبية للتكوين لان هذا الجنس يستطيع ان يتكيف في كافة الافي الاعماق البحرية على اختلافها (Morkhoven, 1963).

\section{الاستنتاجات}

استتادا الى الانتشار الطباقي لاجناس الاوستراكودا تبين بان بيئة ترسيب تكوين كراكو في بئر

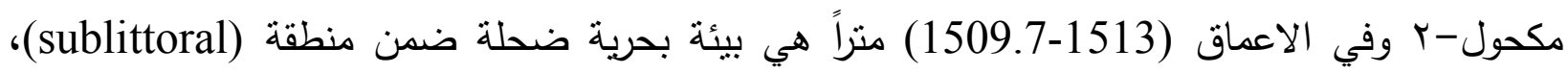

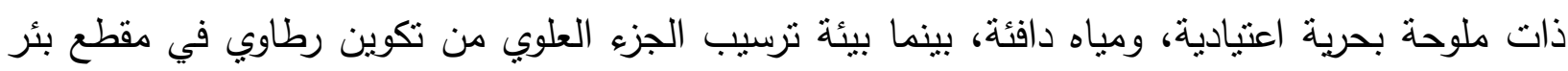

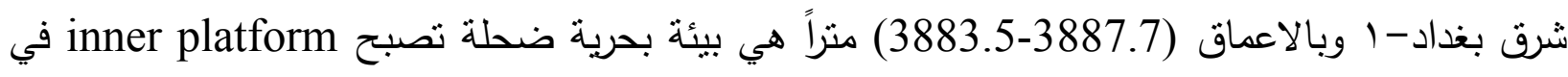

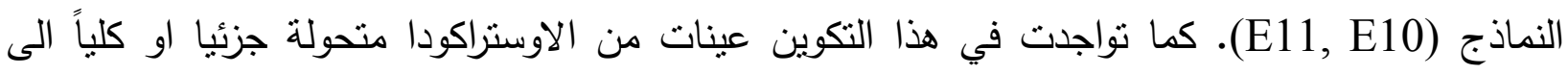

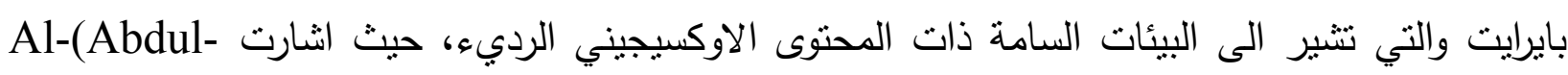
Razzaq, 1983) الثناحب (كما في نماذج تكوين رطاوي) تشير الى تكون البيئات السامة. 


\section{المصادر الأجنبية}

Al-Abdul-Razzaq, S. K. and Grosdidier, E., 1981. Ostracode index species from the Cenomanian of the South shelf of the Tethys Sea. Bull. Cent. Rech. Explor. Prod. Elf. Aquitaine, Vol. 5, pp. 173 - 191.

Al-Abdul-Razzaq, S., 1983. Biostratigraphi zonation of the Amadi Formation (Cretaceous, Kuwait) by using Ostracoda assemblages. in: Applications of Ostracodes, Proc. $8^{\text {th }}$. Ostracod. Symp. Houston, 1982, pp. 394 - 399.

Al-Eisa, M. A. S, 1997.Stratigraphy of Early Cretaceous of Subsurface Sections from Middle Iraq. (Unpub. Ph.D. Thesis), Univ. Mosul, pp.1-195. [in Arabic]

Amin, D. H., 1989. Biostratigraphy of Gragu Limestone Formation in North Iraq. Unpub. M. Sc. Thesis, Baghdad Univ., pp. 1-93.

Andreu, B. and Odin, G. S., 2001. Synthetic data on the Campanian-Maastrichtian ostracode fauna from Tercis les Bains (Landes, Odin G.S. (ed., 2001a, chap. France). In: C6c, pp. 410 - 413.

Andreu, B., 1996. Nouvelles especes d'ostracodes du Turonien Superieur (?)Coniacien (?)-Santonien de la region de Boulmane, Moyen Atlas, Maroc. Systematique, biostratigraphie et paleoecologie, paleobiogeographie des associations. 12e Colloque African de Micropaleontologie, Angers, ElfAquitaine.

Andreu, B., 2001. Ostracodes du site de Tercis les Bains (Landes, France). In: Odin G.S. (ed., 2001a), chap. C6b, pp. 402-409.

Babinot, J. -F. and Bourdillon-de-Grissac, C., 1987. Associations d' Ostracodes de l'Albien-Maastrichtien du Dhofar (Oman), affinities paleobiogeographiques et implications geodynamiques. Bull. Soc. Geol. France, Vol. 5, No. 8, pp. 287 - 294.

Bartenstein, H. and Abawi, T. S., 1984. Lower Cretaceous in Iraq microfacies, stratigraphical subdivision and analysis of the Middle Sarmord Formation in the Kirkuk area. Erdol and Kohle-Erdgas-Petrochemie Vereinigt mit Brennstoff-Chemie. Bd. Vol. 37, pp. 197 - 200.

Bassoullet, J. P. and Damotte, R., 1969. Quelques Ostracodes nouveaux du Cenomano-Turonien de I'Atlas Saharien occidental (Algerie). Rev. Micropaleontol., Vol. 12, pp. 130-144.

Bassoullet, J. P. and Damotte, R., 1969. Quelques Ostracodes nouveaux du Cenomano-Turonien de I'Atlas Saharien occidental (Algerie). Rev. Micropaleontol., Vol. 12, pp. 130 - 144.

Bellen, R. C. Van, Dunnington, H. V., Wetzel, R. and Morton, D., 1959. Lexique Stratigraphique Internal Asie. Iraq. Intern. Geol. Congr. Comm. Stratigr., 3, Fasc. 10a, pp. 1 - 333. 
Breman, E., 1976. Paleoecology and Systematics of Cenomanian and Turonian Ostracoda from Guadalajara and Soria (Central Spain). Rev. Esp. Micropaleont., Vol. 8, pp. 71 - 122.

British Petroleum and Idemitsu, 1990. Bassin Analysis and prospectivity Vol.1, regional geology and stratigraphy. London.

Chatton, M. and Hart, E., 1960. Revision of the Tithonian to Albian of Iraq. IPC Report, No. 1, 141p.

Dall'antonia, B., Bossio, A. and Guernet, C., 2003. The Eocene/Oligocene boundary and the psychrospheric event in the Tethys as recorded by deep-sea ostracoda from the Massignano Global boundary stratotype section and Point, Central Italy. Mar. Micropaleo., Vol. 48, pp. 91-106.

Damotte, R. and Odin, G. S., 2001. Ostracodes du site de Tercis les Bains (Landes, France). In: Odin G.S. (ed., 2001a), chap. C6a, pp. 396 - 401.

Dingle, R. V.,1981. Some Cretaceous ostracodal assemblages from the Agulhas Bank (South-African Continental Margin). Transactions of the Royal Society of South Africa, Cape Town, Vol. 38, No. 4, pp. 393 - 418.

Ditmar, V. Afanasiev, J. and Shankova, E., 1971. Geological conditions and hydrocarbon prospects of the Republic of Iraq (Northern and Central parts). INOC library , Baghdad.

Douban, A. F. and Medhadi, F., 1999. Sequence chronostratigraphy and petroleum system of the Cretaceous Megasequences, Kuwait. AAPG International Conference and Exhibition, Vol. 1, pp. 52 - 155.

Elewa, A. M. T., 2004. Quantitative analysis and palaeoecology of Eocene Ostracoda and benthic foraminifera from Gebel Mokattam, Cairo, Egypt. Palaeogeogr., Palaeoclimatol., Palaeoecol., Vol. 211, pp. 309 - 323.

Ishizaki, K. and Kaiho, K., 1990. Japan in Early Cenomanian Times relative to Tethys and Arctic waters. Saiti Ho-on Kai Spec. Pub., Vol. 3, pp. 177-189.

Jassim, S. Z. and Karim, S. A., 1984. Final report on regional geology survey of Iraq, Vol. 4, Paleogeography, SOC, Baghdad.

Johnson, R. W., 1961. Final well report on Samarra-1. Manuscript report FWR 50, INOC Library, Baghdad.

Kuznetsova, K. I. and Dobrova, M. R., 1995. Endemic and Cosmopolitan Assemblages of Foraminifers and Ostracods from the Middle Jurassic Basins of Syria. Stratigraphy and Geological Correlation, Vol. 3, No. 2, pp. 134 - 146.

Kuznetsova, K. I. and Dobrova, M. R., 1997. Middle Jurassic basins of the Eastern Mediterranean and their microbiota (Foraminifera and Ostracoda). Stratigraphy and Geological Correlation, Vol. 5, No. 1, pp. 29 - 37.

Lazaro, J. R., 1988. Nuevas especies de ostracodos en el Coniaciensey Santoniense de la Cuenca Vasco-Cantabrica. Rev. Esp. Micropaleont., Vol. 20, No. 1, pp. 71 - 107. 


\section{البيئة القيمة لنكوين كراكو والجزء العلوي من تكوين رطاوي (الكريتاسي الاسفل)...................}

Malz, H., Hofmann, K., Radtke, G. and Cherchi, A., 1985. Biostratigraphy of the Middle Jurassic of NW Sardinia by means of ostracods. Senckenbergiana Lethaea, Vol. 66, No. 3/5, pp. 299 - 345.

Mazzini, I., Anadon, P., Barbieri, M., Castorina, F., Ferreli, L., Gliozzi, E., Mola, M. and Vittori, E., 1999. Late Quaternary sea-level changes along the Tyrrenian coast near Orbetello (Tuscany, central Italy): palaeoenvironmental reconstruction using ostracoda. Mar. Micropaleontol., Vol. 37, No. 3-4, pp. $289-311$.

Morkhoven, F. P. C. M. Van, 1962-1963. Post Paleozoic Ostracoda. Elesevier, Amesterdam, Vol. 1, pp. 1-204, Vol. r, pp. 1-478.

Neale, J. W., 1977. Cretaceous Ostracoda of the North Atlantic Basin. In: Swain,

F. M. (ed.), Stratigraphic; Micropaleontology of Atlantic basin and borderlands, Elsevier, pp. 245 - 269.

Neale, J. W., 1978. The Cretaceous. :325-384. In: R. Bate and E. Robinson, E. (eds.), A stratigraphical index of the British Ostracoda. Geol. J. Special Issue No. 8.

Rosenfeld, A. and Raab, M., 1974. Cenomanian-Turonian ostracodes from the Judea Group in Israel (Palestine). Bull. Geol. Surv. Isr., Vol. 62, pp. 1- 64.

Rosenfeld, A., Gerry, E. and Honigstein, A., 1988.Jurassic-Cretaceous nonmarine ostracods from Israel and Palaeoenvironmental implications. In : Hanai, T., Ikeya, N. and Ishizaki, K. (eds.), Evolutionary biology of Ostracoda its fundamentals and applications, Proc. $9^{\text {th }}$ Int. Symp. Ost., Shizuoka, Japan, July/August 1985, Elsevier, pp. 659 - 669.

Sohn, I. G., 1962. The ostracode genus Cytherelloidea a possible indicator of paleotemperature. Geol. Surv. Res., Washington, pp. 144-147.

Swain, F. M., 1982. Marine and brackish water Cretaceous Ostracoda from wells in central and southern Florida. J. Micropalaeontol., Vol. 1, pp. 115-128.

Swain, F. M., 1985 . Some Ostracoda from the Rodessa, Pearsall, Sligo and Upper Hosston Formations (Lower Cretaceous) of Louisiana (U.S.A.). Rev. Micropalaeont., Vol. 27, No. 4, pp. 266 - 294.

Szczechura, J., Abd-Elshafy, E. and Babinot, J. -F., 1991. Late Albian to Early/Mid-Cenomanian Ostracodes from Northern Galala Plateau, Egypt. Acta Palaeont. Polonica, Vol. 36, No. 1, pp. 3 - 33.

Warne, M. T., 1993. Bythocypridiidae (Ostracoda) from the Miocene of the Port Phillip and Western Port Basins, Victoria. Proc. Royal Soc. Victoria, Vol. 102, pp. 105 - 115.

Zarrago, E. G. and Lazaro, J. R., 1990. Late Cretaceous ostracode faunas from the Biscay synclinorium (Basque Arc, Northern, Spain). Cour. Forsch.-Inst. Senckenberg., Vol. 123, pp. 229 - 238. 


$$
\text { سند عبد الإله الخشاب و آخرون }
$$

\section{Plate1}
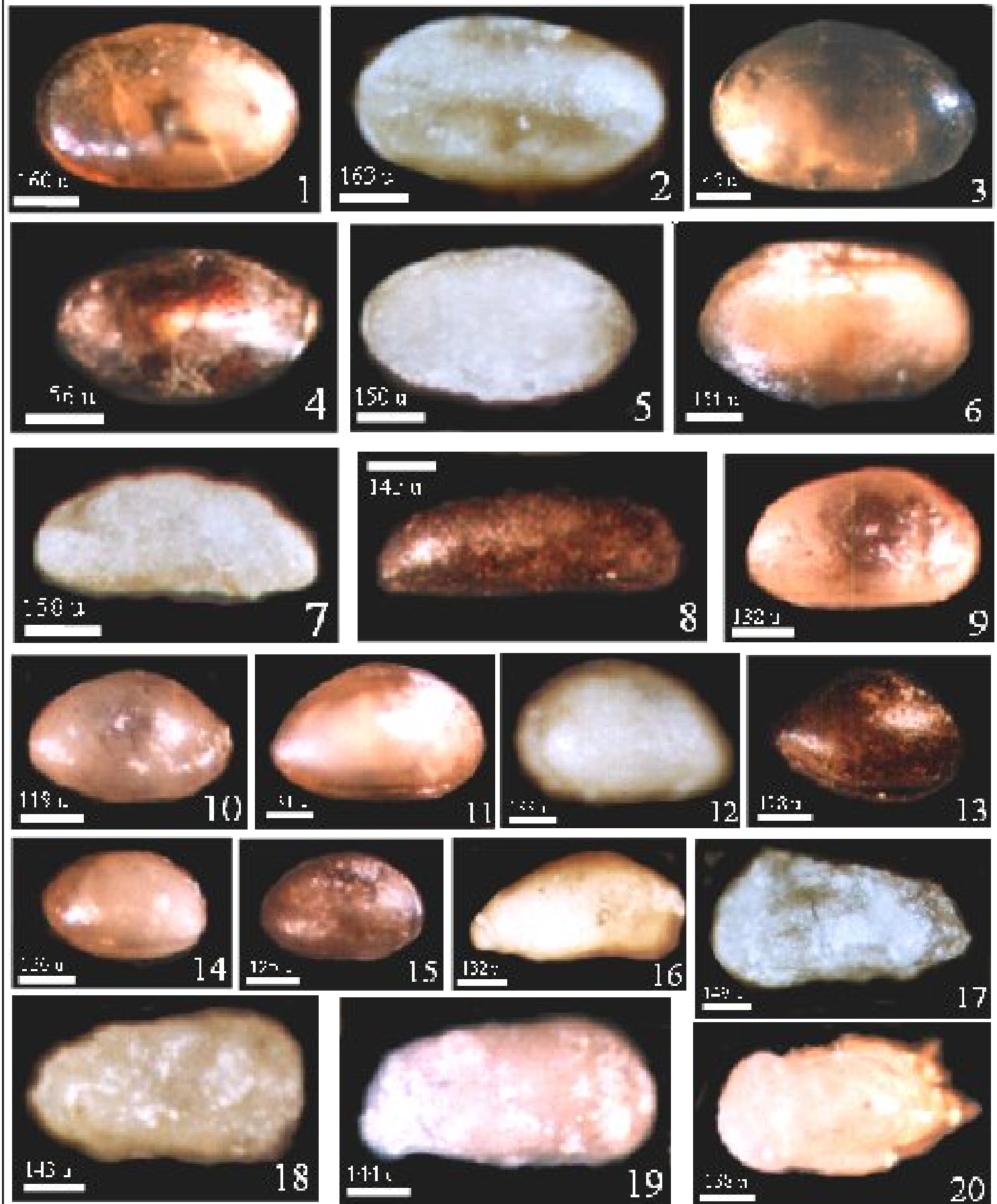

12 1:3:1
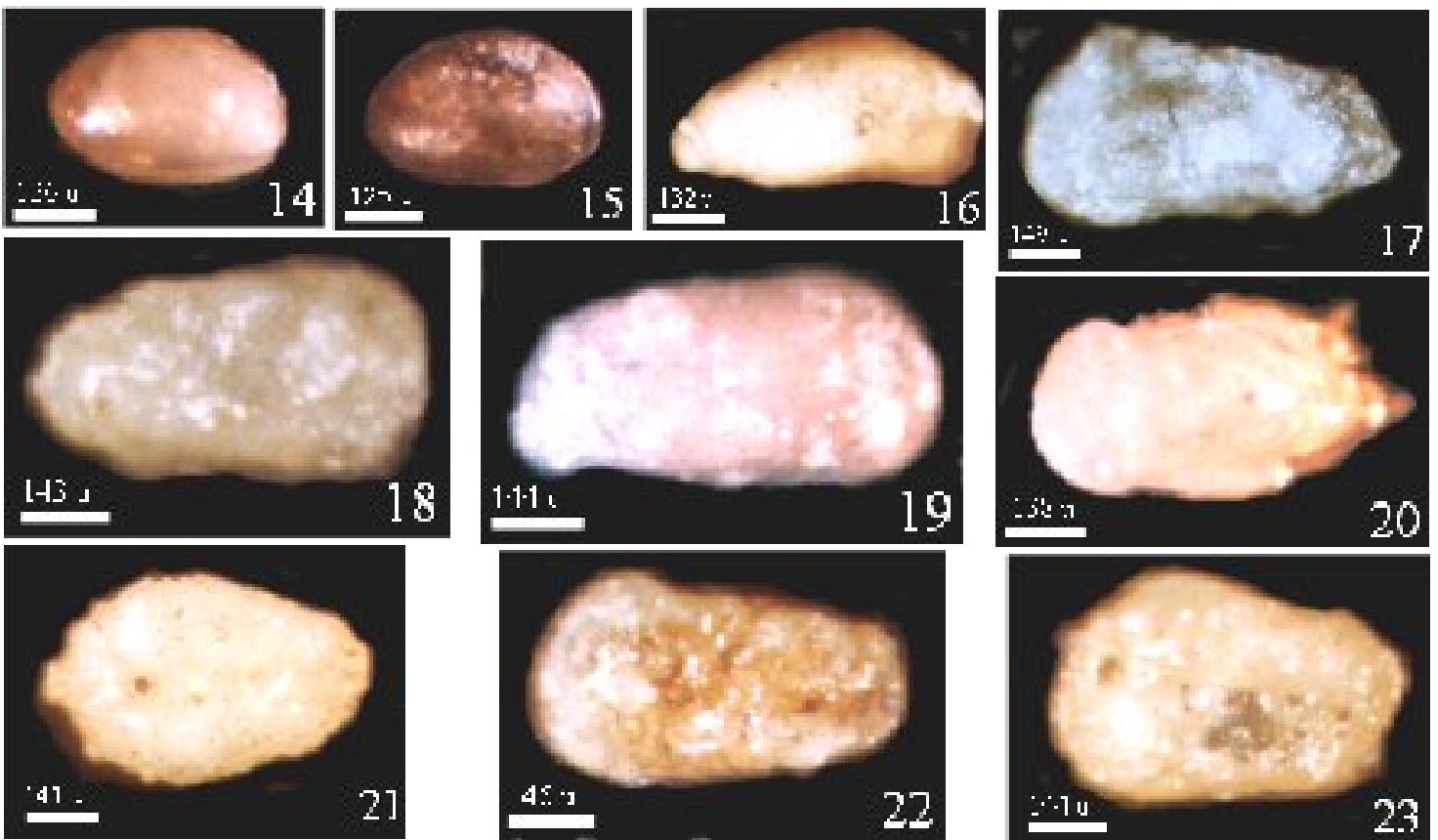

Explanation of Plate1: (1) Cytherella ovata, (2-3) Cytherella posterosulcata, (4) Cytherella cf. sp. OUM1110, (5) Cytherella sp., (6) Cytherella sp. U10, (7) Paracypris? sp., (8) Macrocypris aff. siliqua, (9-10) Asciocythere cf. circumdata, (11) Asciocythere sp.1, (12-13) Schuleridea cf. altera, (14-15) Schuleridea cf. obtabilis, (16) Metacytheropteron? grosdidieri, (17) Cythereis btaterensis btaterensis, (18-20) Cythereis laqueformis, (21) Rehacythereis bernardi, (22) Rehacythereis cf. libanensis, (23) Spinoleberis yotvataensis. 